\title{
Semantic awareness in a nonlexical task
}

\author{
SHLOMO BENTIN \\ Haskins Laboratories, New Haven, Connecticut \\ and Hadassah Medical Center, Jerusalem, Israel
}

and

\author{
LEONARD KATZ \\ Haskins Laboratories, New Haven, Connecticut \\ and University of Connecticut, Storrs, Connecticut
}

\begin{abstract}
Depth of processing of printed words was investigated by comparing priming effects in a task in which subjects made lexical decisions for both the prime and the target with priming effects in a task in which the subjects made "case" decisions for the prime (i.e., decided whether it was written in lower- or uppercase) and lexical decisions on the targets. Thirty-two target words were preceded either by semantic associates or by unrelated words. For both "case" and "lexical decision" conditions for the prime, equal facilitation was found for related targets, suggesting that subjects analyzed words at a semantic level even while making "case" decisions. These results question the applicability of a level-of-processing model for word recognition processes.
\end{abstract}

The present study was concerned with the question of whether a levels-of-processing explanation is appropriate for word recognition and, if so, whether the depth of the processing is under the subject's strategic control. An alternative way of asking the question is: Can a skilled reader perceive a word without accessing its meaning, or are lexical access and semantic processing automatic processes that are triggered by perception of print.

There is general agreement that a word can, in principle, be processed at different levels. This agreement is based primarily on demonstrations that recognition and recall are better for words about which semantic decisions are required than for words about which orthographic or phonemic decisions are required (e.g., Craik \& Lockhart, 1972). It has been assumed that semantic decisions necessitate an additional level of processing beyond that required for orthographic or phonemic decisions, and that this further coding accounts for the superior retention that is found. Consequently, it has been inferred that, in nonlexical tasks, words are processed more superficially, that is, only to the level that provides the information necessary to perform the task. However, a retention test is not an appropriate test for the presence of semantic information that may exist only at the time of encoding. Semantic information

Portions of this study were presented at the 24th annual meeting of the Psychonomic Society, San Diego, CA, November 1983. S. Bentin is with the Aranne Laboratory of Human Psychophysiology at the Hadassah Medical Center. L. Katz is with the Department of Psychology at the University of Connecticut at Storrs. Both authors are associated with Haskins Laboratories, New Haven, CT 06510. may be activated automatically during a word's presentation but, nevertheless, may not reach awareness and, consequently, may not be transferred to a longer term memory. Thus, the memory difference following "semantic" and "nonsemantic" tasks may be accounted for by differences in the quality of the memory trace rather than by differences in the level of processing attained during acquisition (Fisher \& Craik, 1977; Jacoby \& Craik, 1978). Although task requirements may influence the extent to which the word is later remembered, measures of performance other than recall may remain immune to a "depth" manipulation. This was supported by Jacoby and Dallas (1981), who reported that the magnitude of perceptual advantage deriving from prior presentation of a word in a tachistoscopic word recognition task was not affected by the level of task requirement during the prior exposure.

One way to determine whether semantic information is or is not available at the time of encoding is to make use of tasks in which semantic information would clearly affect overt performance if it were present. The investigation of semantic priming in a lexical decision task provides just such an opportunity. It has been repeatedly demonstrated that when two semantically associated words are presented in sequence as stimuli in a lexical decision task, the response time to the first member of such a pair (which is referred to as the "prime") is longer than that to the second (which is referred to as the "target") (e.g., Meyer \& Schvaneveldt, 1971, 1976; Schvaneveldt \& McDonald, 1981). When the orthographic and/or phonemic similarity between the two words has been controlled, we must assume that the facilitation effect on target recognition is related to the prior activation of the semantic field of the prime, 
which includes the target itself. Therefore, no facilitation should be expected if semantic information had not been made available during processing the prime.

Although semantic priming has been extensively investigated, little is known about the relationship between the way in which the prime is processed and the efficiency of the priming effect. A straightforward application of the levels-of-processing model and generalization of the results that were reported in the retention studies cited above would predict that no priming should occur when no semantic decision is required about the prime. Several recently published studies suggest that this is indeed the case. Henik, Friedrich, and Kellogg (1983) reported that recognition of the target was facilitated when the prime was named, but that this effect was reduced to a nonsignificant level when the prime was searched for an individual letter. They concluded that in order to produce the usual priming effect, primes must be processed for meaning. Smith, Theodor, and Franklin (1983) compared the amount of priming in five tasks that were assumed a priori to differ in regard to the depth to which the prime was processed. Priming was absent in a "visual" task in which the subject was instructed to report whether a star was or was not presented to the left of the word and in a "letter-search" task in which the subject was instructed to report whether any of the letters in the word was identical to a letter presented repeatedly above each of the characters in the word string. In contrast, a semantic relationship between target and prime facilitated recognition of the targets when subjects were required to make a phonemic decision about the prime (one syllable or more than one syllable), to silently read the prime without making any specific decision about it, or to make a lexical decision about the prime. The amount of facilitation was largest when a lexical decision was made about the prime and smallest in the phonemic analysis condition.

Although suggestive, these reports do not help us to determine whether, given the opportunity and enough time, subjects are indeed capable of restricting the processing of a word to the minimum required by the task or, on the contrary, whether complete, that is, semantic, coding of words is automatic and obligatory. The difference in the magnitude of the priming effects between the last three conditions in the Smith et al. (1983) study described above, might be explained, for example, by postlexical access processes, but, since no semantic priming is possible without access to the semantic information in the prime, these results suggest that, in these three conditions, semantic information was available to the subject. Apparently, semantic codes were not generated in the visual and in the letter-search tasks. This result challenges the hypothesis that the generation of semantic codes during presentation of words is obligatory. However, in the visual task, the subject's attention may have been shifted away from the priming word due to the spatial location of the star. As for the letter search, as Henik et al (1983) men- tioned, this task required decomposition of the stimulus; when attention was directed to the analysis of individual letters, it may not have been allocated to the word as a whole, and consequently, no whole-word effects (semantic effects) were possible. Therefore, it seems to us that additional examination is required before the "automatic access to meaning" hypothesis can be rejected.

In the present study, a lexical decision was always required on target items, but the type of task performed on the primes was varied. In one task, the subjects were instructed to determine whether the prime was presented in upper- or lowercase characters. This task is orthographic, nonsemantic in nature and does not require lexical access. Note that the decision about the case can be done by analyzing only one letter. However, since the required decision is not in regard to a specific letter in the word string, attention is not necessarily directed to the level of individual letters as it is in the letter-search task. In the second condition, the subjects were instructed to make lexical decisions on both the prime and the target trials. Thus, by definition, processing of the prime in the second condition necessarily included lexical access. The critical comparison between the two conditions was in the amount of target facilitation induced by a prime that was semantically related to the following target.

\section{METHOD}

\section{Stimuli and Design}

From the subject's point of view, each trial consisted of two events: (1) a cue that determined which decision was required for the next stimulus and (2) the stimulus itself. There were 320 such trials presented in four blocks of 80 trials each. From the experimenter's point of view, however, stimuli were presented in pairs, S1 and S2. The 24 subjects were required to make a "case" decision on the S1 trial (i.e., to determine whether the stimulus was written in lower- or uppercase letters) and a "word" decision on the S2 trials (i.e., to determine whether or not the string of letters represented a word). Twenty-four different subjects made lexical decisions on both $\mathrm{S} 1$ and $\mathrm{S} 2$ trials (Figure 1). The time interval between the onset of the cue and the stimulus was kept constant at $1,500 \mathrm{msec}$. However, the intertrial interval (ITI) (i.e., the time from stimulus offset to the onset of the next cue) was $300 \mathrm{msec}$ for half the subjects and $800 \mathrm{msec}$ for the other half. The ITI was varied because we thought that the subjects might use more elaborate semantic coding given the longer time interval and, if so, that we would observe stronger semantic facilitation effects. The subjects in the short stimulus-onset-asynchrony (SOA) and in the long SOA conditions were equally divided among "word-word" and "case-word" groups.

The stimuli were 184 words and 136 nonwords. Among the words, there were 64 pairs of semantic associates and 56 fillers that were either S1 words followed by S2 nonwords or S2 words preceded by $S 1$ nonwords. The 64 pairs of semantic associates were composed of 32 pairs of antonymous adjectives, such as BIG-SMALL, and 32 pairs of nouns related by category, such as SNOW-RAIN. The members in each pair were matched for frequency according to the Kuxera and Francis (1967) list of word frequency. Two different arrangements of the stimuli were prepared: In List $A$, the 2 members of 32 of the 64 related pairs were presented in succession as $\mathrm{S} 1$ and $\mathrm{S} 2$, while the members of the other 32 pairs were scrambled, so that no semantic 

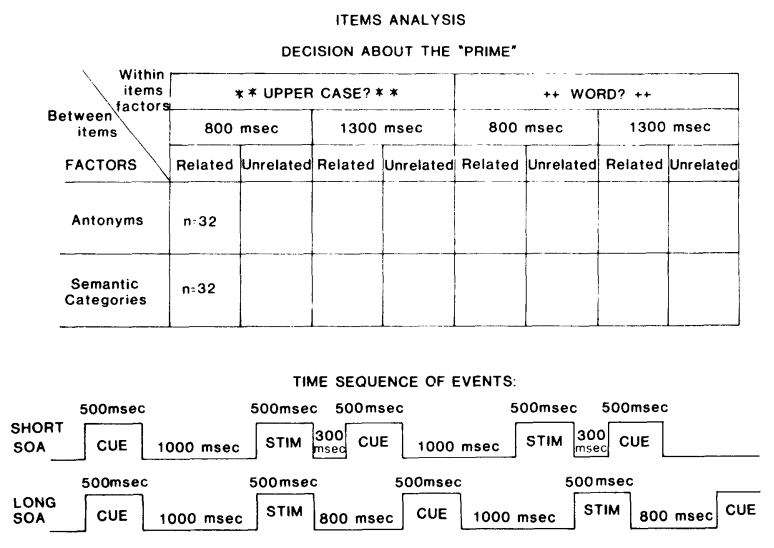

Figure 1. The experimental design and the time sequence of events used in the study.

relation existed between $\mathrm{S} 1$ and $\mathrm{S} 2$. Those items that were unrelated in List A formed the related S1 and S2 in List B, and vice versa. To summarize, the relevant stimuli were 32 pairs of semantically related and 32 pairs of semantically unrelated pairs of words, which were counterbalanced across subjects.

\section{RESULTS}

Only reaction times to $\mathrm{S} 2$, the targets, were analyzed. For the item analysis, the within-items factors were: (1) the decision required at S1 ("case" or "word" decision); (2) SOA (short or long); and (3) semantic relationship between $\mathrm{S} 1$ and $\mathrm{S} 2$ (related or nonrelated). The between-items factor was the type of the relationship (antonym or category membership). For the subjects analysis, there were two between-subjects factors (SOA and decision at $\mathrm{S} 1$ ) and two within-subjects

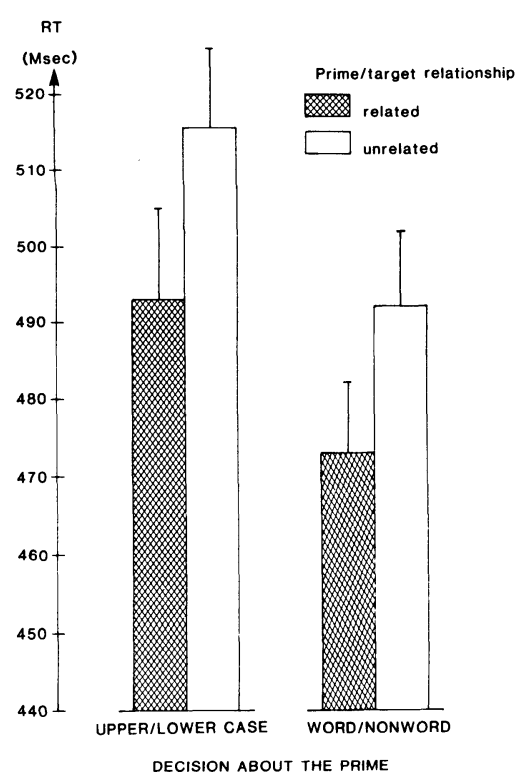

Figure 2. Reaction times to targets related and unrelated to the primes in the "case" and "word" conditions. factors (relationship between S1 and S2 and type of relationship) (Figure 2).

Targets were responded to faster when they followed related primes than when they followed unrelated words. This main effect was significant both for item analysis and for subject analysis $\left[\min F^{\prime}(1,120)=25.23\right.$, $\mathrm{p}<.01]$. All targets were responded to faster when a lexical decision had been required on the previous S1 trial than when an orthographic (case) decision had been required on $\mathrm{S} 1\left[\min \mathrm{F}^{\prime}(1,94)=3.99, \mathrm{p}<.05\right]$. The most important result of this study was that these two factors did not interact: Target recognition was similarly facilitated whether the prime was processed at a socalled "deep" level (lexical decision) or a "shallow" level (case decision).

The SOA factor did not affect performance significantly. However, on the item analysis only, there was a significant interaction between SOA and decision about the prime. When an orthographic decision was made on an S1 trial, the lexical decision on the following S2 trial was faster with long than with short SOAs. By contrast, the SOA did not affect the lexical decision on S2 when a lexical decision was also made about $\mathrm{S} 1$. This interaction was not significant, however, in the subjects analysis.

\section{CONCLUSIONS}

The main result is that similar amounts of target facilitation were found regardless of the decision required about the prime. As such, these results contradict prior studies that have suggested that a "shallow" task induces "shallow" processing of the prime, which produces little semantic facilitation of the target. These results also replicate previous unpublished results obtained in our laboratory for a similar paradigm. It seems, therefore, that the generalization of the levels-of-processing model to word recognition is limited to specific tasks and designs. Our results suggest that, given the time and opportunity, subjects cannot or will not restrict processing of a word to a shallow level. Presentation of a word seems to trigger a process that, if not disturbed, will automatically continue until the stimulus is semantically coded.

\section{REFERENCES}

Craik, F. I. M., \& Lockhart, R. S. (1972). Levels of processing: A framework for memory research. Journal of Verbal Learning and Verbal Behavior, 11, 671-684.

Fisher, A. M., \& Craik, F. I. M. (1977). The interaction between encoding and retrieval operations in cued recall. Journal of Experimental Psychology: Human Learning and Memory, 3, 701-711.

Henik, A., Friedrich, F. J., \& Kellogg, W. A. (1983). The dependence of semantic relatedness effects upon prime processing. Memory \& Cognition, 11, 366-373.

JACOBY, L. L., \& CrAIK, F. I. M. (1978). Effects of elaboration of processing at encoding and retrieval: Trace distinctiveness and recovery of initial context. In L. S. Cermak \& F. I. M. Craik (Eds.), Human memory: A cognitive view. Hillsdale, NJ: Erlbaum.

JACOBY, L. L., \& DALLAs, M. (1981). On the relationship between autobiographical memory and perceptual learning. Journal of Experimental Psychology: General, 3, 306-340.

KučERA, H., \& Francis, W. N. (1967). Computational analysis of present-day American English. Providence, RI: Brown University Press. 
Meyer, D. E., \& Schvaneveldt, R. W. (1971). Facilitation in recognizing pairs of words: Evidence of a dependence between retrieval operations. Journal of Experimental Psychalogy, 90, 227-243.

Meyer, D. E., \& Schvaneveldt, R. W. (1976). Meaning, memory structure and mental processes. Science, 192, 227-243.

Schvaneveldt, R. W., \& McDonald, J. E. (1981). Semantic context and the encoding of words: Evidence of two modes of stimulus analysis. Journal of Experimental Psychology: Human Perception and Performance, 7, 673-687.

Smith, M. C., Theodor, L., \& Franklin, P. E. (1983). On the relationship between contextual facilitation and depth of processing. Journal of Experimental Psychology: Learning, Memory, and Cognition, 4, 697-712.

(Manuscript received for publication May 8, 1984.) 